\title{
Comprehensive Analysis of Strip- and Slot-Line Guided Forward, Backward, and Complex Magnetostatic Waves
}

\author{
Rachid Rafii-El-Idrissi, Ricardo Marqués, Member, IEEE, and Francisco Medina, Member, IEEE
}

\begin{abstract}
This paper presents a comprehensive and accurate analysis of the guidance of volume and surface magnetostatic waves by strips or slots in the presence of a ferrite slab magnetized to saturation by an external uniform magnetic field. The strip/slot can be directly printed on the ferrite surface or separated from it by means of a dielectric layer of arbitrary thickness. The problem is posed in terms of a suitable integral equation accounting for the magnetostatic limit. This equation is solved by using a spectral-domain formulation. The proposed method allows for the consideration of arbitrary magnetization angles and the presence of upper and/or lower ground planes. Strip-guided forward, backward, and complex surface magnetostatic waves have been obtained and analyzed in terms of the appropriate waveguide parameters. Slot-guided backward volume magnetostatic waves have been also computed and studied. Theoretical results have been checked against theoretical (magnetostatic and full wave) and experimental data available in the literature with reasonable agreement.
\end{abstract}

Index Terms-Microstrip line, slot line, magnetostatic waves, ferrites.

\section{INTRODUCTION}

G UIDED magnetostatic waves (MSWs) are useful in the design of delay lines, filters, couplers, and other microwave magnetostatic devices [1]. YIG films produced by epitaxial growth and other planar structures are widely used for this purpose. The propagation of MSWs along unbound slab ferrite loaded waveguides is a classical topic today (see, e.g., [2]). In such an analysis, the field variation along the direction perpendicular to propagation and parallel to the ferrite film is neglected. However, any practical waveguide must have finite dimensions. Thus, propagation along ferrite films of finite width is analyzed in [3]. Other kind of MSW guides of finite width are reported in [4]. An important class of finite MSW waveguides are those obtained by placing metallic strips or slots over the ferrite substrate. These waveguides strongly confine the electromagnetic field in the region below the strip or around the slot and can be easily manufactured by using well-known technologies. Strip- and/or slot-guided MSWs are also of interest in the analysis of dynamic microwave ferrimagnetic devices, such as isolators or phase shifters, since

Manuscript received July 28, 2000. This work was supported by the Comisión Interministerial de Ciencia y Tecnología, Spain under Project TIC98-0630 and by Junta de Andalucía.

The authors are with the Microwaves Group, Department of Electronics and Electromagnetism, School of Physics, University of Seville, 41012 Seville,

Spain (e-mail: marques@cica.es; medina@cica.es).

Publisher Item Identifier S 0018-9480(01)07585-8. guided MSWs may appear, at some frequencies, in addition to dynamic modes, giving rise to relevant effects [5]-[7].

To the authors knowledge, magnetostatic surface waves (MSSWs) guided by metallic strips printed on a ferrite slab were first reported and analyzed in [8]. Experimental results on the propagation of MSSWs along suspended strips were reported in [9]. Although no specific analytical or numerical calculations were carried out in that paper, the presence of backward modes in those structures was predicted in [9] from the physical interpretation of the experimental results. Backward-volume magnetostatic waves (BVMSWs) guided by a metallic slot printed on a ferrite substrate have been recently reported in [10]. Forward-volume magnetostatic waves (FVMSWs) guided by a metallic strip over a ferrite YIG film with nonuniform magnetization perpendicular to the plane of the substrate have been reported in [11], in which a simplified magnetic-wall model was used. Nevertheless, if the magnetization is uniform, it should not be ignored that the propagation of FVMSWs along those structures is leaky: FVMSWs are excited in the ferrite substrate which propagate normally to the strip direction. This fact is experimentally demonstrated in [12], in which it is also shown that the radiation losses due to that mechanism are small for wide strips. Although this paper is restricted to the study of bound modes (leaky modes are not computed), our analytical method makes apparent that all these strip-guided FWMSWs are leaky.

In [13], the authors analyzed the propagation of MSSWs along ferrite loaded strip lines. Forward, backward, and complex bound modes were reported in [13] for the appropriate frequencies and waveguide parameters. Such kind of modes were previously reported for unbound ferrite loaded parallel-plate waveguides by Marqués et al. [14]. In addition, the presence of bound complex modes in nonreciprocal waveguides as an alternative to evanescent reactive modes of the reciprocal ones was demonstrated in [15]. The integral spectral domain analysis (ISDA), previously used in [13], will be extended in this paper to analyze MSWs guided by metallic strips or slots printed on a ferrite substrate or on a dielectric slab backed by a ferrite substrate. The ferrite material is assumed magnetized to saturation by an externally applied arbitrarily oriented uniform static magnetic field. Note that the proposed ISDA technique can account for any direction of the internal magnetization, as well as for any distance from the strip/slot to the ferrite slab. The presence of one or two upper/lower ground plane(s) is also accounted for. This analysis must be accompanied by the analysis of the various slab waveguides 


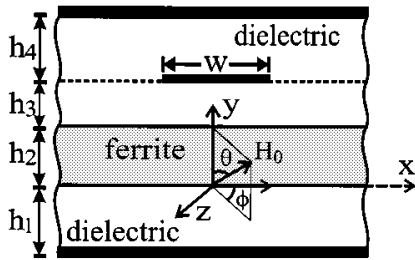

a)

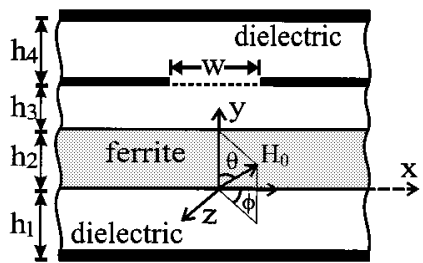

b)
Fig. 1. Waveguides under study. (a) Ferrite loaded strip line. (b) Ferrite loaded slot line.

in which the ferrite/dielectric loaded strip or slot line can be divided. Such analysis is fundamental in order to establish the magnetization and frequency ranges of existence of the studied strip- and slot-guided MSWs. In this way, the mechanisms of guidance of the different types of strip-/slot-guided MSWs are described, and the existence of these waves predicted before any numerical computation is performed. Once the range of existence is known, the exact influence of the strip or slot width is accounted for by means of the ISDA in the frame of the magnetostatic approach.

\section{SPECTRAL-DOMAIN ANALYSIS}

Although the ISDA is a well-known technique for the analysis of strip-/slot-like structures [16], the application of this technique to magnetostatic waveguides presents some special features deserving discussion. The structures under analysis are shown in Fig. 1(a) and (b). A metallic strip [see Fig. 1(a)] or slot [see Fig. 1(b)] is placed over a ferrite slab magnetized to saturation (a dielectric layer can be present between the strip/slot and the ferrite slab). Upper and/or lower ground(s) plane(s) can be present and the direction of the internal bias magnetic field is arbitrary. An implicit field dependence of the kind $\exp j\left(\omega t-k_{z} z\right)$ is assumed in the following. Let us define a current function $I(x)$ at each point $x$ along the strip/slot interface as follows:

$$
I(x)=\int_{-\infty}^{x} J_{s, z}\left(x^{\prime}\right) d x^{\prime}
$$

where $J_{s, z}$ is the surface current density on this interface. Since only MSWs will be analyzed, this current function must vanish at $x= \pm w / 2$ (strip or slot configurations) and at $x= \pm \infty$ (slot configuration). The magnetic field is then derived from the magnetostatic potential $\psi$ as $\mathbf{H}=-\nabla \psi$ and $\mathbf{B}=\bar{\mu} \cdot \mathbf{H}$, where $\bar{\mu}$ is the magnetic permeability tensor. For saturated ferrites, $\bar{\mu}$ is the Polder tensor, whose expression for an arbitrary direction of the internal magnetization can be found in many textbooks (see, e.g., [2 App. C]).

In order to obtain an implicit equation for the propagation constant $k_{z}$ as a function of the angular frequency $\omega$, integral equations have to be derived for the current function (strip configuration case) $I\left(x, k_{z}, \omega\right)$ or the $y$-component of the magnetic flux density at the slot interface (slot configuration case) $B_{y}\left(x, k_{z}, \omega\right)$. Fortunately, a simple algebraic linear relation among those quantities can be written in the $k_{x}$ Fourier transform domain

$$
\tilde{B}_{y}\left(k_{x}, k_{z}, \omega\right)=\tilde{G}\left(k_{x}, k_{z}, \omega\right) \tilde{I}\left(k_{x}, k_{z}, \omega\right)
$$

where $k_{x}$ is the Fourier variable associated to the $x$-Fourier transform, $(\cdot)$ signifies the $x$-Fourier transform, and $\tilde{G}\left(k_{x}, k_{z}, \omega\right)$ is a suitable spectral-domain Green's function whose expression can be derived in closed form in terms of the constitutive and geometrical parameters of the layered structure (see the Appendix).

The relation given in (2) can be translated into the space domain, after applying the inverse Fourier transform, giving the following two linear convolutions:

$$
B_{y}\left(x, k_{z}, \omega\right)=\int_{-\infty}^{\infty} G_{B}\left(x-x^{\prime}, k_{z}, \omega\right) I\left(x^{\prime}, k_{z}, \omega\right) d x^{\prime}
$$

and

$$
I\left(x, k_{z}, \omega\right)=\int_{-\infty}^{\infty} G_{I}\left(x-x^{\prime}, k_{z}, \omega\right) B_{y}\left(x^{\prime}, k_{z}, \omega\right) d x^{\prime}
$$

where $G_{B}\left(x-x^{\prime}, k_{z}, \omega\right)$ is the spatial-domain counterpart of $\tilde{G}\left(k_{x}, k_{z}, \omega\right)$ in (2) and $G_{I}\left(x-x^{\prime}, k_{z}, \omega\right)$ is the the space-domain version of $\left[\tilde{G}\left(k_{x}, k_{z}, \omega\right)\right]^{-1}$. After imposing the boundary conditions $B_{y}(x)=0$ on the strip $(-w / 2<x<w / 2)$ and $I(x)=0$ outside the strip $(|x|>w / 2)$, (3) is an integral equation for $I(x)$, which yields an implicit equation for $k_{z}(\omega)$ in the strip-line case [see Fig. 1(a)]. Conversely, (4) together with the conditions $I(x)=0$ on the slot $(-w / 2<x<w / 2)$ and $B_{y}(x)=0$ outside the slot $(|x|>w / 2)$ gives an integral equation for $B_{y}(x)$ from whose resolution an implicit equation for $k_{z}$ is obtained [see slot line case in Fig. 1(b)]. The integral equations (3) and (4) for the strip and slot configurations are readily solved using the Galerkin method in the spectral domain [16], with subsectional triangular basis functions for both $B_{y}(x)$ and $I(x)$.

In order to predict the regions of existence of the bound guided MSWs of interest in this paper, it is also necessary to know the dispersion and equiphase curves of the modes of the various slab or parallel-plate waveguides in which the strip- or the slot-line configurations can be split. These slab or parallel-plate waveguides are obtained by taking the limits $w=0$ and/or $w \rightarrow \infty$ in Fig. 1. Those dispersion curves have been obtained by using the method described in [14] for the dynamic analysis of layered structures, after adaptation to the magnetostatic case. It is worth noting that the magnetostatic modes of the waveguides in Fig. 1(a) and (b) with $w=0$ coincide with the poles (zeroes) of the spectral Green's function in (2). In particular, an MSW mode propagating in the aforementioned waveguide with propagation constant in the $x$-z-plane $\mathbf{k}=k_{x, n} \hat{\mathbf{x}}+k_{z, n} \hat{\mathbf{z}}$ implies the existence of a pole (zero) of $\tilde{G}\left(k_{x}, k_{z}, \omega\right)$ at $k_{x}=k_{x, n}$ and $k_{z}=k_{z, n}$. Following the analysis in [17] and [18], when the phase constant of a given bound mode of the strip (slot) waveguide of Fig. 1(a) and (b) reaches a value $k_{z, m}$, which is compatible with the propagation of a slab mode in the region further the strip (slot) zone (i.e., when $k_{z, m}=k_{z, n}$ ), the strip mode (slot mode) becomes leaky. This fact will be used below to establish the range of existence of bound strip-guided (slot-guided) magnetostatic modes.

\section{MSSWs GUIDED By METALLIC STRIPS}

Although the propagation of MSSWs along strip lines has been studied elsewhere [8], a brief description of the mecha- 


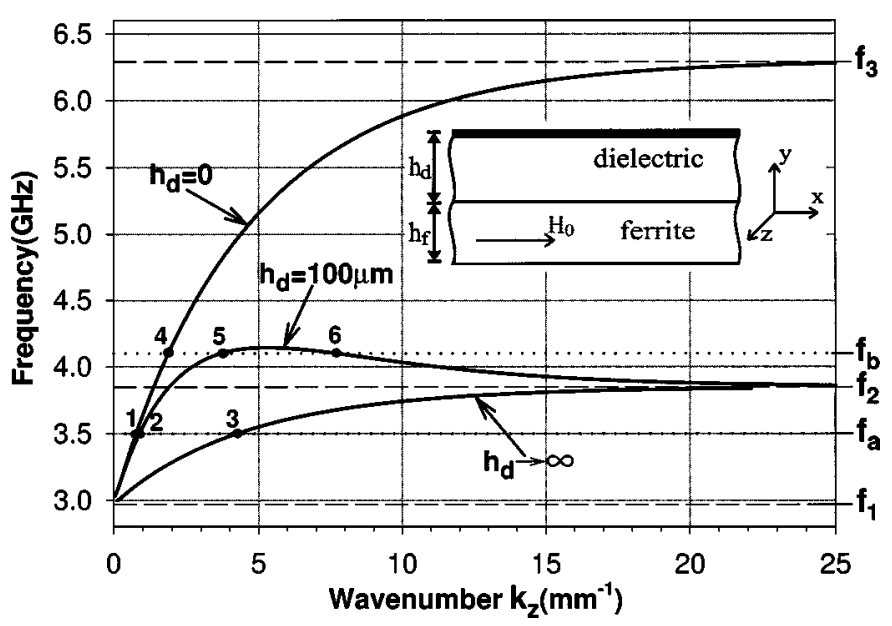

Fig. 2. Propagation characteristics of MSSWs along a metallized ferrite slab. Structure parameters: $h_{f}=100 \mu \mathrm{m}, 4 \pi M_{s}=1750 \mathrm{G}, H_{0}=500 \mathrm{Oe}$. The dielectric constant is irrelevant for MSW propagation.

nism of guidance is given here for the sake of completeness. It is a well-known fact that a ferrite-air or ferrite-metal interface with a magnetization parallel to this interface can support unidirectional MSSWs propagating in the direction defined by the cross product $\mathbf{H}_{0} \times \hat{\mathbf{n}}$, where $\mathbf{H}_{0}$ is the static magnetic biasing field and $\hat{\mathbf{n}}$ is the unit vector normal to the interface and directed outwards the ferrite medium [2].

In a more realistic structure, such as a ferrite slab, the frequency range of excitation for MSSWs - which are obviously bidirectional due to the symmetry of the structure-is $f_{1}<$ $f<f_{2}$, where $f_{1}=\sqrt{f_{0}\left(f_{0}+f_{M}\right)}, f_{2}=f_{0}+(1 / 2) f_{M}$ and $2 \pi f_{0}=\gamma H_{0}$, with $2 \pi f_{M}=\gamma\left(4 \pi M_{s}\right), \gamma$ being the gyromagnetic ratio and $M_{s}$ being the saturation magnetization of the ferrite [2]. MSSWs can be also excited along a ferrite slab having one of its faces metallized. The frequency range of excitation is, in such cases, $f_{1}<f<f_{3}$, where $f_{3}=f_{0}+f_{M}$. Since the structure is not symmetrical with respect to a horizontal plane, these MSSWs will not be bidirectional [2].

Some representative dispersion curves are shown in Fig. 2 for a grounded ferrite slab (see the inset of Fig. 2). Three cases are considered: $h_{d} \rightarrow \infty$ (nonmetallized or free slab), $h_{d}=0$ (conductor backed ferrite slab), and the intermediate situation $0<h_{d}<\infty$. Propagation is assumed to be in the positive $z$-direction, i.e., parallel to $\mathbf{H}_{0} \times \hat{\mathbf{y}}$, and $k_{x}$ is assumed to be zero. Let us examine more closely the situation. Fig. 3(a) and (b) shows the equiphase curves in the $k_{x}-k_{z}$-plane for two particular frequencies, i.e., $f_{a}$ and $f_{b}$, in the ranges $f_{1}<f_{a}<f_{2}$ and $f_{2}<f_{b}<f_{3}$, respectively (see $f_{a}$ and $f_{b}$ in Fig. 2). Note that there is a range of values of $k_{z}$ for which there is propagation in the conductor-backed ferrite structure and there is no possibility of propagation along the free slab structure. In general, for a couple of values of $h_{d}$, there is always a range of $k_{z}$ values for which propagation is possible along the structure with the smaller value of $h_{d}$, but it is not possible along the structure with the larger value of $h_{d}$. Note that this situation is just the opposite of the situation encountered if only simple dielectric materials were present (i.e., if ferrites were removed). With regard to the waveguide of Fig. 1(a), if, for instance, $f=f_{a}, h_{3}=0, h_{4}=h_{1}=\infty$, this implies that

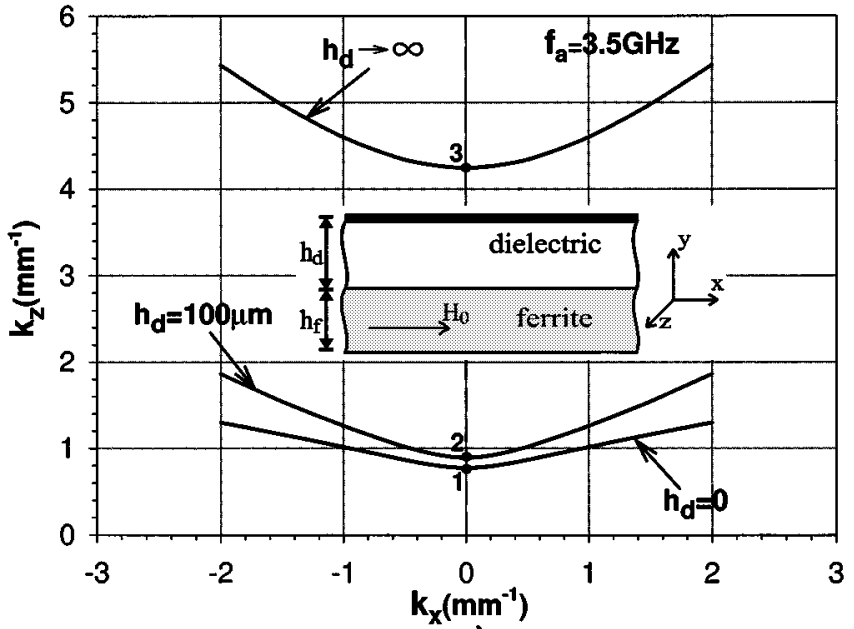

a)

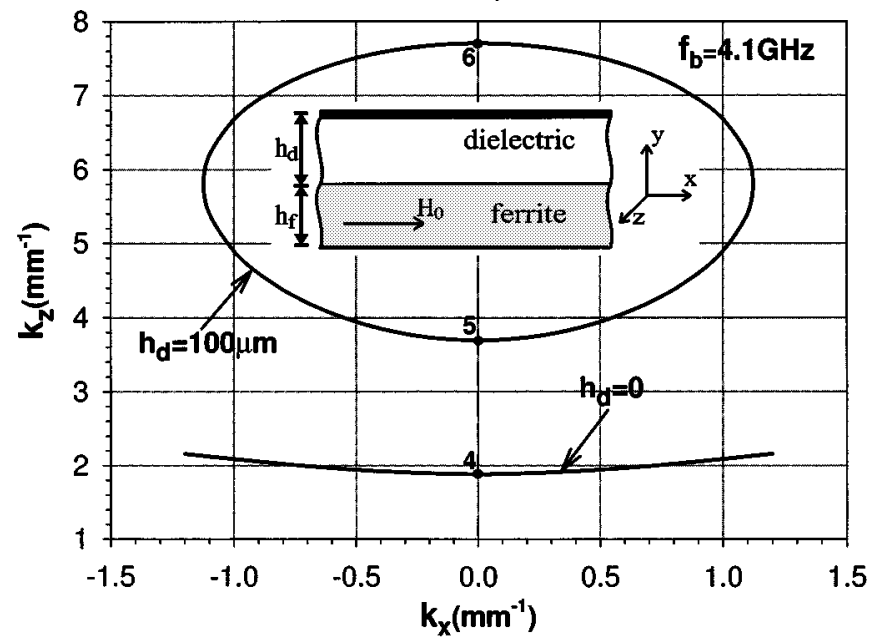

b)

Fig. 3. (a) Equiphase curves for the MSSWs in Fig. 2 at the frequency $f_{a}=$ $3.5 \mathrm{GHz}$. (b) Equiphase curves for the MSSWs in Fig. 2 at the frequency $f_{b}=$ $4.1 \mathrm{GHz}$.

there is a range of $k_{z}$ values for which propagation of MSSWs in the region below the strip is possible, but not in the zone outside the strip [this zone ranges between the points marked 1 and 3 in Fig. 3(a)]. Since guided modes can be viewed as the result of the reflections at the strip edges of two-plane MSSWs propagating in the region below the strip with the same $k_{z}$ and opposite values of $k_{x}$, this fact suggest that MSSWs can be guided by the strip-line structure shown in Fig. 1(a) with $\mathbf{H}_{0}=H_{0} \hat{\mathbf{x}}$. Since propagation along conductor-backed ferrite slab waveguides is unidirectional, strip-guided MSSWs will be also unidirectional. After extending the above analysis to all frequencies, these waves are expected to appear in the region of the $k_{z}-\omega$-plane limited by the dispersion curves of the waveguides obtained from the structure in Fig. 1(a) in the two limit cases $w=0$ (surface $y=h_{2}+h_{3}$ is not metallized) and $w \rightarrow \infty$ (surface $y=h_{2}+h_{3}$ is metallized). In addition, from a more detailed inspection of the curves in Fig. 2, we can see that, for finite nonzero values of $h_{d}$ and for frequencies in the $f_{2}<f<f_{3}$ range, backward surface waves are possible in the metal-dielectric-ferrite geometry. This fact results in the existence of closed curves in Fig. 3(b). This 


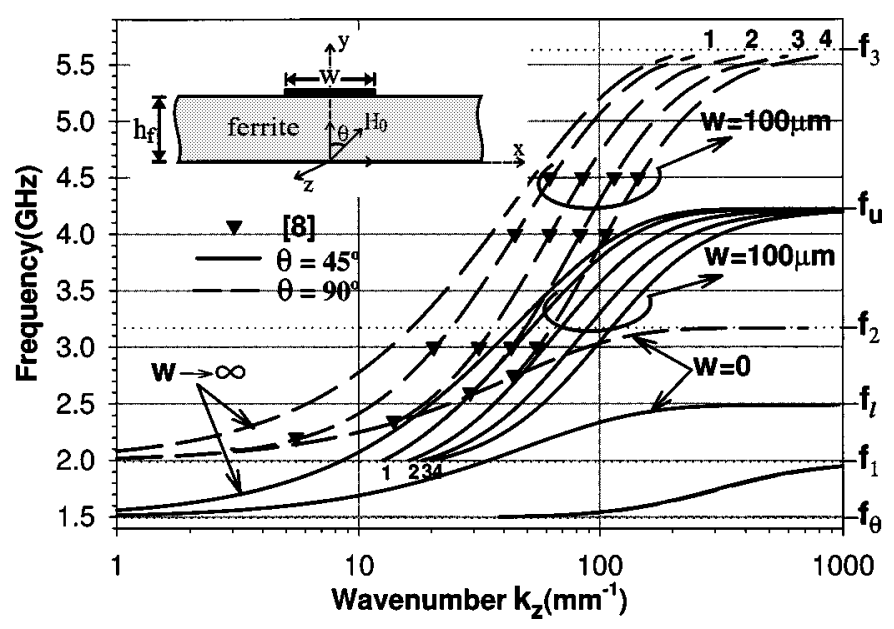

Fig. 4. Dispersion curves for the first four bound MSSW modes of the strip line on a ferrite slab shown in the inset. Structure parameters: $h_{f}=10 \mu \mathrm{m}$, $w=100 \mu \mathrm{m}, 4 \pi M_{s}=1760 \mathrm{G}, H_{0}=251 \mathrm{Oe}, \theta=45^{\circ}, 90^{\circ}$. The first MSSW modes and the first two FVMSW modes of the metallized $(w=0)$ and nonmetallized $(w \rightarrow \infty)$ ferrite slabs are also shown. Comparison with the results in $[8]\left(\theta=90^{\circ}\right)$ is also shown.

peculiar situation suggests the possibility of having backward or even complex modes guided by the strip line in Fig. 1(a) provided $h_{3} \neq 0$. This speculative idea will be confirmed later with numerical results for that type of guided modes.

The propagation of MSSWs along strip lines was first demonstrated in [8], in which MSSW propagation along an infinite array of equally spaced strips printed over a ferrite slab with magnetization parallel to the interface and perpendicular to the strips was analyzed. The case of a single isolated strip was considered in [8] by increasing the separation between the strips until numerical convergence was reached. The method used in this paper allows us to deal with more general structures. Since it has no restrictions on the direction of the static magnetizing field, it can account for the presence of a dielectric region between the strip and ferrite layer and upper and/or lower ground plane(s) can be considered. In order to illustrate the accuracy of this method, we have compared in [13] our magnetostatic results with those obtained following the method reported in [7], where a relatively cumbersome full-wave analysis was applied. An excellent agreement between magnetostatic and full-wave results has been found, proving the accuracy of the present method. It is worth mentioning that the numerical effort required by the method in this paper is much lower than that required by the full-wave formulation in [7]. In addition, the analysis reported here provides a deep physical insight than cannot be extracted from the blind analysis carried out in [7].

In [8], it was shown that an MSSW guided by metallic strips printed on a ferrite film form an infinite, but discrete, set of unidirectional modes with increasing values of the phase constant $k_{z}$. On the other hand, [13] shows that the phase constant increases when the strip width decreases. The same is true for the time delay. The effect of the magnetization angle is analyzed in Fig. 4, where dispersion curves for the first four MSSWs corresponding to two different orientations of the magnetic field in the plane perpendicular to the strip

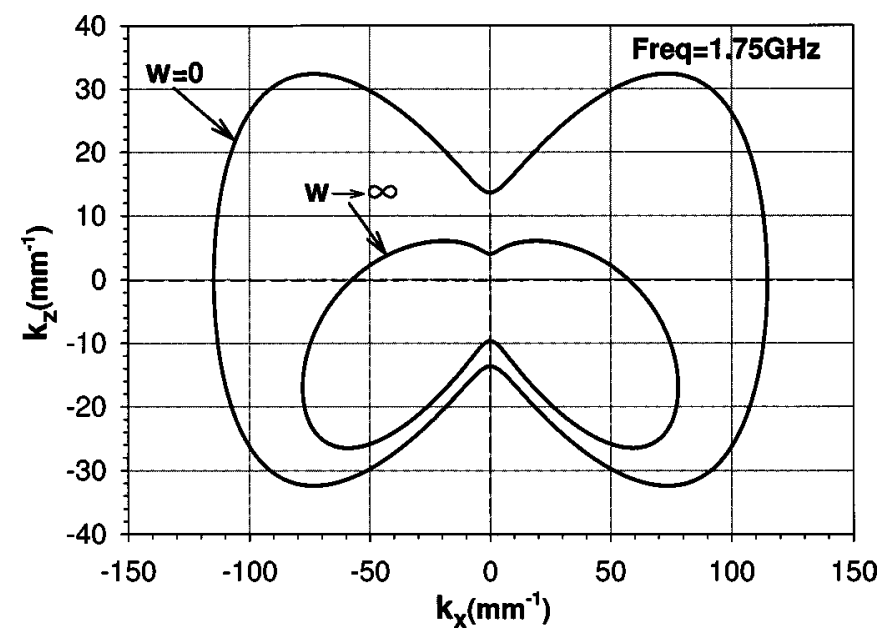

Fig. 5. Equiphase curves for the first FVMSW modes of the metallized ( $w \rightarrow$ $\infty)$ and nonmetallized $(w=0)$ ferrite slab of Fig. 4 at the frequency $f=$ $1.75 \mathrm{GHz}$.

$\left(\theta=90^{\circ}\right.$ and $\left.\theta=45^{\circ}, \phi=0\right)$ are depicted. For comparison purposes, data in [8] for the $\theta=90^{\circ}$ case are included. The dispersion curves of the metallized $(w \rightarrow \infty)$ and the unmetallized $(w=0)$ ferrite slab magnetostatic modes are also shown. Strip-guided MSSW modes appear in the region of the $k_{z}-\omega$-plane comprised between the dispersion curves of the MSSW modes of the metallized and unmetallized slab, and above the frequency line $f=f_{1}=\sqrt{f_{0}\left(f_{0}+f_{M}\right)}$. Below this frequency, the strip modes become leaky due to the excitation of FVMSWs in the ferrite slab. Notice that an effect of the variation of the internal bias field orientation is the reduction of the frequency range of excitation of the strip-guided MSSWs. This reduction occurs because the upper frequency limit for the excitation of MSSWs (in both the metallized and unmetallized ferrite slab) is also reduced, as can be seen in Fig. 4, where the upper frequency limit $f_{u}$ is below the upper limit $f_{3}=f_{0}+f_{M}=5.63 \mathrm{GHz}$ for horizontal magnetization $\left(\theta=90^{\circ}\right)$. Another effect is the important increasing of the $k_{z}$ wavenumber at a fixed frequency.

FVMSWs excited in the slab waveguides below the strip $(w \rightarrow \infty)$ and outside the strip region $(w=0)$ are also shown in Fig. 4. They appear in the frequency range $f_{\theta}<f<f_{1}$, with $f_{\theta}=\sqrt{f_{0}\left(f_{0}+f_{M} \sin ^{2} \theta\right)}$ [2], [19]. The dispersion curves of the first FVMSW modes are the continuation of the dispersion curves of the slab MSSW modes for the frequencies $f<f_{1}$ [19]. The dispersion curves for the second slab FVMSW modes corresponding to both the metallized and the nonmetallized structures are also shown in Fig. 4. These curves are practically indistinguishable on the graph. The equiphase curves of the first FVMSW modes (metallized and nonmetallized ferrite slab) at a frequency within the range $f_{\theta}<f<f_{1}(f=1.75$ $\mathrm{GHz})$ are depicted in Fig. 5. These are closed curves around the origin $\left(k_{x}=k_{z}=0\right)$. The equiphase curves for the higher order FVMSWs show a similar behavior, but with larger values of $k_{z}$ and $k_{x}$. This topology is very general and has been observed in FVMSWs for a wide range of frequencies and internal magnetization angles. It must be noted that arbitrary variations of the azimuth angle $\phi$ of the internal magnetizing 


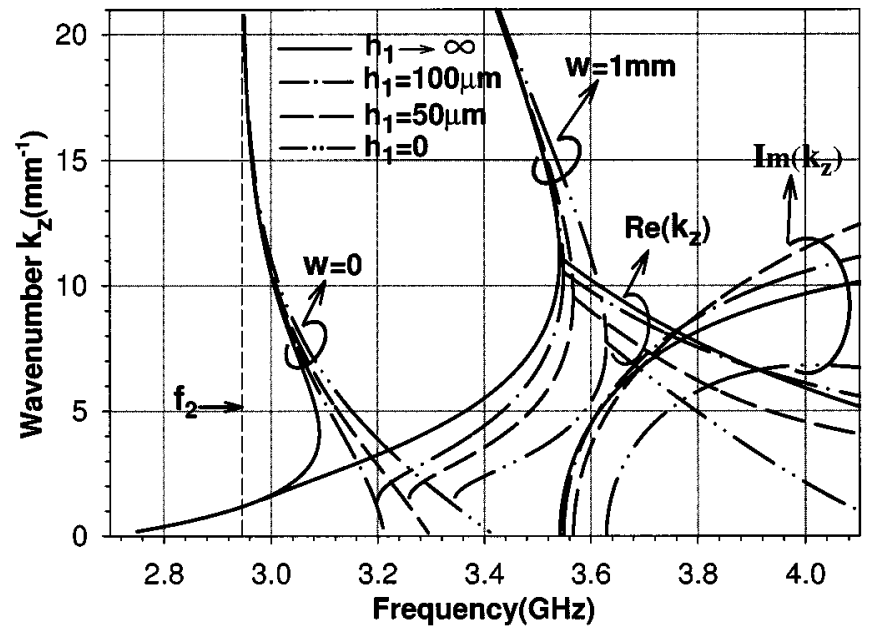

Fig. 6. Dispersion curves for the first MSSW modes of the strip line of Fig. 1(a) with $h_{2}=100 \mu \mathrm{m}, h_{3}=20 \mu \mathrm{m}, h_{4}=10 \mu \mathrm{m}, w=1 \mathrm{~mm}, 4 \pi M_{s}=840 \mathrm{G}$, $H_{0}=632 \mathrm{Oe}, \theta=90^{\circ}, \phi=0^{\circ}$.

field only imply rotations around the origin- $k_{x}=k_{z}=0$ - of the curves in Fig. 5. Therefore, strip guided FVMSWs, if any, must be leaky due to the excitation of other FVMSWs propagating in the slab and having the same value of $k_{z}$. However, although no bound FVMSW modes have been found in strip lines, leaky FVMSWs may be present [12]. These leaky modes will leak power because of the excitation of the first and higher order FVMSW in the region outside the strip. Since in this paper we have restricted ourselves to the analysis of bound modes, the leaky wave regions will be excluded from the analysis.

The effects of the thickness of the dielectric region between the strip and ferrite slab, as well as the effect of the presence of top/bottom ground plane(s) are sketched in Fig. 6. In this figure, the dispersion curves for the first MSSW mode guided by the structure in Fig. 1(a) with finite values of $h_{1}, h_{3}$, and $h_{4}$ are shown. As mentioned before, strip-guided MSSWs appear in the region of the $\omega-k_{z}$-plane limited, at the lower end of the frequency spectrum, by the dispersion curve corresponding to the waveguide of Fig. 1(a) in the limit case $w=0$ (empty waveguide). These limit curves for several values of $h_{1}$ are included in Fig. 6. At frequencies below such limit curves, the strip-guided MSSW modes become leaky due to the excitation of MSSWs into the empty waveguide at both sides of the strip. Forward and backward modes appear at frequencies $f>f_{2}$ (see Fig. 2). These modes combine becoming complex at some frequency between $f_{2}$ and $f_{3}$ (see Fig. 2). These complex modes are bound modes (i.e., the wave attenuation is not related to radiation losses, but to the reactive nature of the complex mode [20]). This fact might be rather surprising because bound complex modes are unusual in open waveguides. Nevertheless, bound complex modes have been already reported in open waveguides [21]. Complex modes in ferrite-filled parallel-plate waveguides have been also found in [14]. Moreover, it has been shown [15] that all nonbidirectional reactive modes in gyrotropic waveguides must be also complex. All these facts support the physical meaning of the reported MSSW bound complex modes.

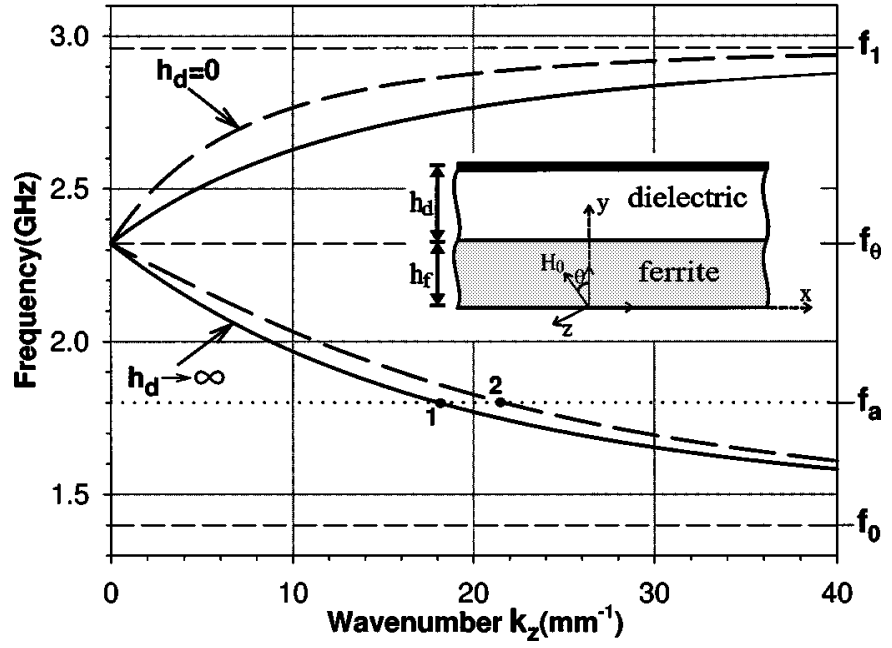

Fig. 7. Dispersion curves for the forward and backward magnetostatic modes propagating in the slab waveguide shown in the inset. Structure parameters: $h_{f}=100 \mu \mathrm{m}, 4 \pi M_{s}=1750 \mathrm{G}, H_{0}=500 \mathrm{Oe}, \theta=45^{\circ}$. Solid lines: free nonmetallized ferrite slab. Dash lines: metal backed ferrite slab.

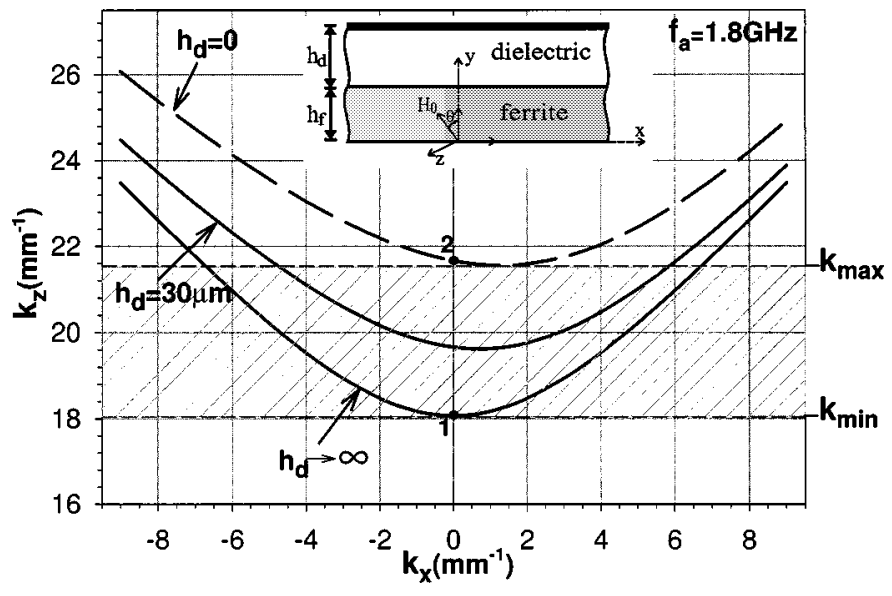

Fig. 8. Equiphase curves for the slab waveguide shown in Fig. 7 at $f=$ $1.8 \mathrm{GHz}$.

\section{BVMSW GUIDED BY SLOT LINES}

Forward- and backward-volume waves can be excited in ferrite slabs at frequencies in the range $f_{0}<f<f_{1}$ [2]. If the biasing magnetic field is perpendicular to the slab, MSWs are forward waves, whereas if this field is parallel to the direction of propagation, MSWs are backward. For a magnetizing field arbitrarily oriented $[\theta$ arbitrary in Fig. 1(b)] in the plane parallel to both perpendicular and longitudinal directions $[\phi=$ $90^{\circ}$ in Fig. 1(b)], both FVMSWs and BVMSWs are excited. BVMSWs appear at frequencies in the range $f_{0}<f<f_{\theta}$, with $f_{\theta}=\sqrt{f_{0}\left(f_{0}+f_{M} \sin ^{2} \theta\right)}$, whereas FVMSWs appear for $f_{\theta}<f<f_{1}$ [2], [22].

In Fig. 7, the dispersion curves for the first MSW mode propagating in free and grounded ferrite slabs (see the inset in this figure) are shown ( $k_{x}=0$ in Fig. 7). Higher order MSWs appear for higher values of $k_{z}$, giving place to an infinite, but discrete, set of modes for each value of $h$. The equiphase curves in the $k_{x}-k_{z}$-plane of the BVMSWs of Fig. 7 for the intermediate frequency $f_{a}=1.8 \mathrm{GHz}$ are shown in Fig. 8 . The equiphase curve for a finite (nonzero) value of $h, h=30 \mu \mathrm{m}$ is also included in Fig. 8. This figure shows how the equiphase curve for 


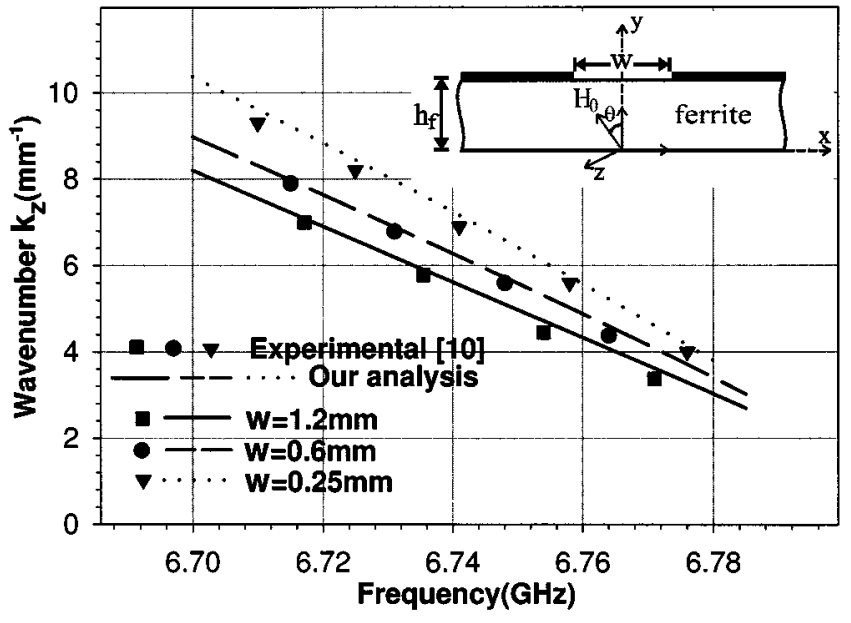

Fig. 9. Dispersion curves for the BVMSW modes propagating along the slot line of Fig. 1(b) with $h_{3}=0, h_{1}=h_{4} \rightarrow \infty, h_{2}=18.7 \mu \mathrm{m}, w=$ $1.2,0.6,0.25 \mathrm{~mm}, 4 \pi M_{s}=1750 \mathrm{G}, H_{0}=1712.5 \mathrm{Oe}, \theta=90^{\circ}, \phi=90^{\circ}$. Symbols show the experimental results reported in [10].

the first BVMSW mode of the structure with the smaller value of $h_{d}$ is above the equiphase curve of the same mode in the structure with the higher value of $h_{d}$. With respect to the waveguide shown in Fig. 1(b), this means that there is a range of values of $k_{z}$ for which BVMSWs can propagate in the region of the slot, but cannot propagate in the region outside the slot (metallized region). This fact suggest that BVMSWs can be guided by the slot structure of Fig. 1(b) with phase constants within the aforementioned range of $k_{z}$ values. The slot-guided BVMSWs can be viewed as the result of the superposition of two-plane BVMSWs traveling along the slot region with the same value of $k_{z}$ and values of $k_{x}^{ \pm}$of the opposite sign. These plane waves would be generated by reflection on the slot edges. For magnetization in the plane $\phi=90^{\circ}$ [see Fig. 1(b)], all the propagating BVMSW modes in the slot waveguide are bidirectional, a fact that was demonstrated in [23] for any gyrotropic waveguide magnetized in that plane. For a given frequency, the permitted values of the phase constant are limited by the maximum and minimum values of $k_{z}$ in the equiphase curves for the waveguide of Fig. 1(b) in the two limits $w \rightarrow \infty$ (nonmetallized surface) and $w=0$ (fully metallized surface). For a printed slot-line $\left(h_{3}=0, h_{4} \rightarrow \infty\right)$ at the frequency $f_{a}=1.8 \mathrm{GHz}$, these values are marked as $k_{\max }$ and $k_{\min }$ in Fig. 8. It should be noted that since the equiphase curves in Fig. 8 are not symmetrical, these values will not coincide, in general, with the phase constants of the corresponding slab waveguides shown in Fig. 7 and marked 1 and 2 in Figs. 9 and 10.

The equiphase curves in the $k_{x}-k_{z}$-plane of the FVMSWs guided by free and metallized ferrite slabs at frequencies $f>f_{\theta}$ are closed curves encircling the origin $k_{x}=k_{z}=0$. Higher order FVMSW modes are also present, and they also give place to closed curves with larger values of $k_{x}$ and $k_{z}$. This behavior is similar to those described in Fig. 5 and, due to this reason, is not explicitly shown here. From this fact, it becomes apparent that, as was shown for strip lines, a bound FVMSW cannot be guided by slot waveguides, although leaky FVMSW modes may be present.

Vulgalter et al. [10] have recently analyzed the total reflection of BVMSWs at a metallic edge over a saturated ferrite slab magnetized in the direction parallel to the edge. From the

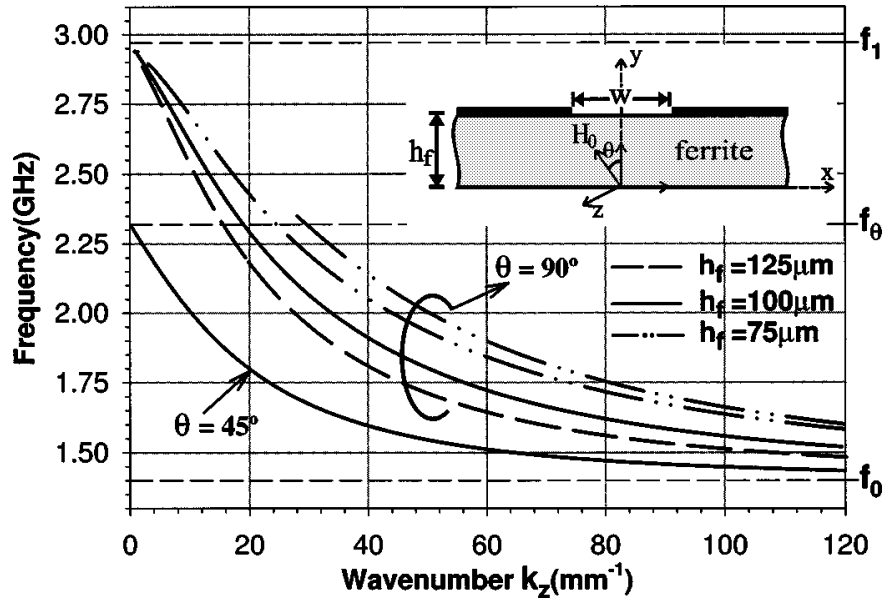

Fig. 10. Dispersion curves for the BVMSW modes propagating in the slot line shown in the inset. The structure parameters are: $w=300 \mu \mathrm{m}, 4 \pi M_{\mathcal{s}}=$ $1750 \mathrm{G}, H_{0}=500 \mathrm{Oe}$.

computed values of the reflection coefficient, they deduced the dispersion curves of wide slot lines on axially magnetized ferrite slabs. Experimental results are also provided in [10]. In Fig. 9, our results, obtained following the method reported in this paper, are compared with the experimental data presented in [10]. The saturation magnetization of the ferrite is $4 \pi M_{s}=$ $1750 \mathrm{G}$, and the value of the static bias field $H_{0}$ is obtained from the experimental value of the antiresonant frequency $f_{1}=$ $\sqrt{f_{0}\left(f_{0}+f_{M}\right)}=6817 \mathrm{MHz}$, which gives $H_{0}=1712.5 \mathrm{Oe}$. The agreement between our computed data and experimental data from [10] is very good, as can be seen from Fig. 9. It should be emphasized that the method presented in this paper is not subjected to the restrictions in [10], being able to deal with arbitrary values of the slot width, as well as arbitrary orientation of the biasing magnetic field in the $y-z$-plane. In Fig. 10, the propagation of BVMSWs along slot lines printed over a ferrite slab magnetized along the $\phi=90^{\circ}$ plane at an arbitrary angle $\theta$ is analyzed. Propagation of a slot-guided BVMSW is expected to occur in the aforementioned frequency range $f_{0}<f<f_{\theta}$ with $f_{\theta}=\sqrt{f_{0}\left(f_{0}+f_{M} \sin ^{2} \theta\right)}$. This results in a reduction of the frequency range of excitation with respect to the $\mathbf{H}_{\mathbf{0}}=H_{0} \hat{\mathbf{z}}$ case $\left(\theta=90^{\circ}\right)$. In Fig. 10, the slot width has been chosen so as to select only one propagating BVMSW mode along the slot line for the $h=100 \mu \mathrm{m}$ case. The number of propagating BVMSW modes that can be excited along the slot line increases with the slot width since the number of permitted values of the transverse wavenumber $k_{x}$ for stationary waves along the $x$-direction also increases. The effect of the ferrite film thickness on the BVMSW phase constant is also shown in Fig. 10. As it is expected from the volume nature of the wave, this effect is important. It can be seen that the phase constant increases when the ferrite film thickness decreases. It can be also observed that, for a given slot width, the number of guided BVMSW modes increases when the ferrite film thickness decreases. This effect can be explained by considering the equiphase curves behavior in Fig. 8. Since $k_{z}$ increases (also for the slab waveguides) when the slab thickness decreases, the number of allowed values of $k_{x}$ also increases, resulting in the excitation of a higher number of modes. The second mode for $h_{2}=75 \mu \mathrm{m}$ in Fig. 10 reach 
the maximum value available for the phase constant $k_{\max }$ (see Fig. 8) at a frequency of $2.36 \mathrm{GHz}$, becoming leaky at this point. Due to this reason, its dispersion curve is finished sharply at that frequency.

\section{CONCLUSION}

A comprehensive analysis of the guidance of MSWs by strips or slots in a layered structure including an arbitrarily magnetized (to saturation) ferrite slab has been presented in this paper. A numerical analysis based on the ISDA/magnetostatic approach has been preceded by a theoretical qualitative study based on the inspection of the features of the elementary slab or parallel-plate waveguides into which the strip or slot guide can be divided. The main conclusions of the paper can be summarized as follows:

- MSSWs (non leaky) - forward, backward, and complex - can be guided by strip lines;

- BVMSWs can be guided by slot lines;

- it is expected that, in most cases, strip/slot guided FVMSWs radiate into the surrounding waveguide, thus being leaky modes.

The main features of the strip-guided MSSWs are as follows:

- unidirectionality;

- existence of an infinite number of modes;

- presence of backward and complex modes when a dielectric layer is placed between the strip and ferrite slab;

- for a certain frequency range, MSSWs guided by the strip are the unique existing magnetostatic modes in the whole structure, including the surrounding ferrite slab waveguide;

- phase constant and delay time can be increased by decreasing the strip width.

The main features of the slot guided BVMSWs are as follows:

- bidirectionality (for $\phi=90^{\circ}$ );

- existence of a finite number of modes;

- number of modes increases if the slot width increases;

- number of modes increases if the height of the ferrite slab decreases.

\section{APPENDIX}

The spectral-domain Green's function in (2) is given by

$$
\tilde{G}\left(k_{x}, k_{z}, \omega\right)=-\mu_{0} k_{d} \frac{A+B}{C+D}
$$

where

$$
\begin{aligned}
A= & \left(\mu_{y, y} k_{f} k_{d} \operatorname{coth}\left(k_{f} h_{2}\right)+\mu_{y, y}^{2} Z \operatorname{coth}\left(k_{d} h_{1}\right)+\mu_{y, y} k_{d} X\right) \\
& \cdot \operatorname{coth}\left(k_{d} h_{3}\right) \\
B= & \mu_{y, y} k_{f} k_{d} \operatorname{coth}\left(k_{f} h_{2}\right) \operatorname{coth}\left(k_{d} h_{1}\right)+k_{d}^{2}-\mu_{y, y} k_{d} X \\
& \cdot \operatorname{coth}\left(k_{d} h_{1}\right) \\
C= & {\left[\mu_{y, y} k_{f} k_{d} \operatorname{coth}\left(k_{f} h_{2}\right)\left(\operatorname{coth}\left(k_{d} h_{1}\right)+\operatorname{coth}\left(k_{d} h_{4}\right)\right)\right.} \\
& +k_{d}^{2}+\mu_{y, y}^{2} Z \operatorname{coth}\left(k_{d} h_{1}\right) \operatorname{coth}\left(k_{d} h_{4}\right) \\
& \left.+\mu_{y, y} k_{d} X\left(\operatorname{coth}\left(k_{d} h_{4}\right)-\operatorname{coth}\left(k_{d} h_{1}\right)\right)\right] \operatorname{coth}\left(k_{d} h_{3}\right)
\end{aligned}
$$

$$
\begin{aligned}
D= & \mu_{y, y} k_{f} k_{d} \operatorname{coth}\left(k_{f} h_{2}\right)\left(1+\operatorname{coth}\left(k_{d} h_{1}\right) \operatorname{coth}\left(k_{d} h_{4}\right)\right) \\
& +k_{d}^{2} \operatorname{coth}\left(k_{d} h_{4}\right)+\mu_{y, y}^{2} Z \operatorname{coth}\left(k_{d} h_{1}\right) \\
& +\mu_{y, y} k_{d} X\left(1+\operatorname{coth}\left(k_{d} h_{1}\right) \operatorname{coth}\left(k_{d} h_{4}\right)\right)
\end{aligned}
$$

with

$$
\begin{aligned}
X & =j \frac{k_{x}\left(\mu_{y, x}-\mu_{x, y}\right)+k_{z}\left(\mu_{y, z}-\mu_{z, y}\right)}{2 \mu_{y, y}} \\
Z & =X^{2}+k_{f}^{2} \\
k_{f} & =\sqrt{S-I^{2}} \\
k_{d} & =\sqrt{k_{x}^{2}+k_{z}^{2}}
\end{aligned}
$$

where

$$
\begin{gathered}
S=\frac{k_{x}^{2} \mu_{x, x}+k_{z}^{2} \mu_{z, z}+k_{z} k_{z}\left(\mu_{x, z}+\mu_{z, x}\right)}{\mu_{y, y}} \\
I=\frac{k_{x}\left(\mu_{x, y}+\mu_{y, x}\right)+k_{z}\left(\mu_{y, z}+\mu_{z, y}\right)}{2 \mu_{y, y}}
\end{gathered}
$$

where $\mu_{x, x}, \mu_{x, y} \ldots$ are the terms of the relative permeability tensor of the ferrite.

\section{REFERENCES}

[1] W. S. Ishak, "Magnetostatic wave technology: A review," Proc. IEEE, vol. 76, pp. 171-187, Feb. 1988.

[2] M. S. Sodha and N. C. Srivastava, Microwave Propagation in Ferrimagnetics. New York: Plenum, 1981

[3] T. W. O'Keefe and R. W. Patterson, "Magnetostatic wave propagation in finite samples," J. Appl. Phys., vol. 49, no. 9, pp. 4886-4895, Sept. 1978.

[4] I. V. Vasil'ev and S. I. Kovalev, "Electrodynamic theory of finite magnetostatic waveguides," IEEE Trans. Microwave Theory Tech., vol. 42, pp. 1238-1245, July 1994.

[5] S. H. Talisa and D. M. Bolle, "On the modeling of the edge-guided mode stripline isolators," IEEE Trans. Microwave Theory Tech., vol. 27, pp. 584-591, June 1979.

[6] _ , "The edge-guided mode nonreciprocal phase shifter," IEEE Trans. Microwave Theory Tech., vol. 27, pp. 878-882, Nov. 1979.

[7] F. Mesa, R. Marqués, and M. Horno, "An efficient numerical spectral domain method to analyze a large class of nonreciprocal planar transmission lines," IEEE Trans. Microwave Theory Tech., vol. 40, pp. 1630-1641, Aug. 1992.

[8] M. Uehara, K. Yashiro, and S. Ohkawa, "Guided magnetostatic surface waves on a metallic strip line," J. Appl. Phys., vol. 54, no. 5, pp. 2582-2587, May 1983.

[9] V. I. Zubkov, E. G. Lokk, and V. I. Scheglov, "Propagation of surface magnetostatic waves under a metal strip placed over a ferrite film," Sov. J. Commun. Technol. Electron., vol. 35, no. 4, pp. 5-8, 1990.

[10] G. A. Vulgalter and A. G. Korovin, "Total internal reflection of backward volume magnetostatic waves and its application for waveguides in ferrite films," J. Phys. D, Appl. Phys., vol. 31, pp. 1309-1319, 1998.

[11] K. Okubo, V. Priye, and M. Tsutsumi, "A new magnetostatic wave delay line using YIG film,” IEEE Trans. Magn., vol. 33, pp. 2338-2341, May 1997.

[12] M. Tsutsumi, T. Ueda, and K. Okubo, "Nonlinear behavior of electromagnetic waves in the YIG film microstrip line," in IEEE MTT-S Int. Microwave Symp. Dig., June 1998, pp. 841-844.

[13] R. Rafii-El-Idrissi, R. Marqués, and F. Medina, "Efficient analysis of magnetostatic surface waves in printed and suspended ferrite loaded strip lines," IEEE Microwave Guided Wave Lett., to be published.

[14] R. Marqués, F. Mesa, and M. Horno, "Nonreciprocal and reciprocal complex and backward waves in parallel plate waveguides loaded with a ferrite slab arbitrarily magnetized," IEEE Trans. Microwave Theory Tech., vol. 41, pp. 1409-1418, Aug. 1993.

[15] — , "On the complex nature of higher order modes in lossless nonreciprocal transversely magnetized waveguides," IEEE Microwave Guided Wave Lett., vol. 2, July 1992.

[16] D. Mirshekar-Syahkal, Spectral Domain Method for Microwave Integrated Circuits. New York: Wiley, 1990. 
[17] N. Das and D. Pozar, "Full-wave spectral domain computation of material, radiation, and guided wave losses in finite multilayer printed transmission lines," IEEE Trans. Microwave Theory Tech., vol. 39, pp. 54-63, Jan. 1991

[18] F. Mesa, R. Marqués, and M. Horno, "Complete modal spectrum of nonreciprocal multilayered microstrip lines," in Proc. URSI Electromag. Theory Symp., Sydney, N.S.W., Australia, Aug. 1992, pp. 397-399.

[19] N. D. J. Miller, "Non-reciprocal magnetostatic volume waves," IEEE Trans. Magn., vol. MAG-14, pp. 829-831, Sept. 1978.

[20] M. Mrozowski, Guided Electromagnetic Waves. Properties and Analysis. New York: Res. Stud. Press, 1997.

[21] T. F. Jablonski, "Complex modes in open dielectric waveguides," J. Opt. Soc. Amer. A, Opt. Image Sci., vol. 11, no. 4, pp. 1272-1282, Apr. 1994.

[22] S. N. Bajpai and N. C. Shrivastava, "Magnetostatic bulk waves in an arbitrarily magnetized YIG-dielectric layered structure," Phys. Stat. Solidi, vol. 57, pp. 307-315, 1980.

[23] V. Dimitriev, "Comments on 'Properties of and generalized full-wave transmission line model for hybrid (bi)(an)isotropic waveguide', "IEEE Trans. Microwave Theory Tech., vol. 47, pp. 655-657, May 1999.

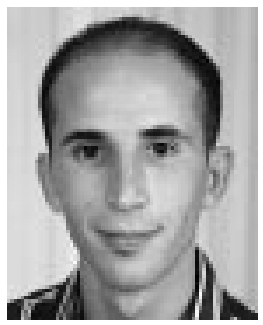

Rachid Rafii-El-Idrissi was born in Meknes, Morocco, in July 1971. He received the Licenciado degree in physics from the University of Meknes, Meknes, Morocco, in 1995, and is currently working toward the Ph.D. in physics at the University of Seville, Seville, Spain

$\mathrm{He}$ is currently with the Microwaves Group, University of Seville. His research interests involves wave propagation in microwave-integrated-circuit (MIC) ferrite devices.

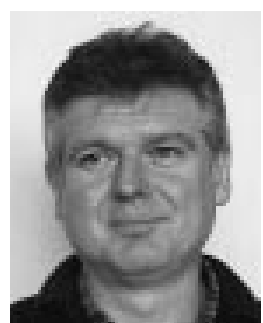

Ricardo Marqués (M'95) was born in San Fernando, Cádiz, Spain. He received the Licenciado and Doctor degrees from the University of Seville, Seville, Spain, in 1983 and 1987, respectively, both in physics.

Since 1984, he has been with the Department of Electronics and Electromagnetism, University of Seville, where he is currently Associate Professor. His main fields of interest include computer-aided design (CAD) for MIC devices, wave propagation in ferrites, and other complex anisotropic and/or bi-isotropic media and field theory.

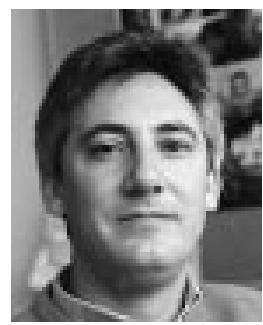

Francisco Medina (M'90) was born in Puerto Real, Cádiz, Spain, in November 1960. He received the Licenciado and Doctor degrees from the University of Seville, Seville, Spain, in 1983 and 1987, respectively, both in physics.

From 1986 to 1987, he was with the Laboratoire de Microondes de l'ENSEEIHT, Toulouse, France. From 1985 to 1989, he was a Profesor Ayudante with the Department of Electronics and Electromagnetism, University of Seville. Since 1990 he has been Profesor Titular of electromagnetism at the University of Seville. He is also currently the Head of the Microwaves Group, University of Seville. His research interests include analytical and numerical methods for planar structures and circuits and the influence on these circuits of anisotropic materials.

Dr. Medina was a member of the Technical Program Committee (TPC) of the 23rd European Microwave Conference, Madrid, Spain (1993) and of the Spanish TPC of the ISRAMT Conference, Málaga, Spain (1999). He is an Editorial Board member of the IEEE TRANSACTIONS ON MICROWAVE THEORY AND TECHNIQUES. He is also a reviewer for other IEEE and Institution of Electrical Engineers (IEE), U.K., journals. He was the recipient of a Ministerio de Educación y Ciencia/Ministere de la Recherche et la Technologie Scholarship. 